\title{
Basement Membrane Coverage Measurement
}

National Cancer Institute

\section{Source}

National Cancer Institute. Basement Membrane Coverage Measurement. NCI

Thesaurus. Code C114791.

The determination of the percentage of a blood vessel that is comprised of intact basement membrane structures. 